\title{
EL PROBLEMA DEL CAMBIO, DESDE LA DIACRONÍA AL SISTEMISMO
}

\author{
Alfredo Martínez
}

(Columbia University)

Plantear la problemática inherente al cambio lingüístico en unas pocas páginas no es en absoluto sencillo. El cultivo de la lingüística histórica, que se remonta a mediados del siglo pasado, ha conocido desde los comienzos del estructuralismo una época de fecundos desarrollos que afectan tanto al fenómeno del cambio como a sus problemas metodológicos (cf. Coseriu, 1958: 8r). Las propuestas de los neogramáticos, los diferentes enfoques estructuralistas, y ciertas implicaciones de las gramáticas generativas (Bynon, 1977: 33-34t) deberían complementarse para intentar un seguimiento de esta cuestión. Pero lo que con las páginas siguientes pretendemos es señalar las principales vías de desarrollo de las que el paradigma saussureano ha sido objeto en la perspectiva estructural, que además de haber ejercido una influencia decisiva sobre la semiótica literaria resulta fundamental para comprender fenómenos inherentes al cambio, como la pareja recurrente de tradición e innovación.

1. Formación de la perspectiva diacrónica. Tras el cultivo secular del descriptivismo lingüístico, desde Grecia a Port Royal, el siglo XIX supuso el despertar de la conciencia histórica. En un ambiente cultural dominado por el evolucionismo y el tradicionalismo histórico, la lingüística buscó la explicación de las lenguas en sus orígenes y desarrollos, dejando a 
un lado las descripciones lógico-gramaticales que hasta entonces habían imperado. La lingüística histórica y la neogramática están en la base de la formación de la perspectiva diacrónica que como tal surgiría en la escuela de Ginebra.

El fenómeno de la novedad lingüística, considerado como la aparición dentro de una lengua de elementos no pre-existentes, se engloba en la categoría superior del cambio. Es el cambio, la modificación, lo que provoca, sobre el eje temporal, la aparición y desaparición de los rasgos lingüísticos, lo cual puede presumiblemente dar razón de la conformación real de una lengua en un momento determinado. Se disciernen entonces con suma claridad dos metodologías diferentes pero emparentadas: la explicación mediante la descripción de un sistema real y la explicación mediante la descripción de su formación. La ruptura epistemológica que esta separación produjo llegó a ser interpretada como la división de la lingüística en dos ciencias inconexas, al menos hasta la superación estructuralista del hiato.

Saussure fue quien con más fortuna explicitó estas divergencias, aunque ya con anterioridad era evidente la doble perspectiva de análisis; recuérdese la distinción humboldtiana entre las dimensiones dinámica y estática de la lengua (energeia, fuerza activa, y ergon, producto). Pero la reformulación saussureana en términos de sincronia y diacronía no supuso sólo una novedad terminológica, sino que implicó consecuencias teóricas y epistemológicas.

En primer lugar, como ha mostrado Engler (1988), la historia de la lengua que practicaban los neogramáticos no es equivalente a la diacronía de Saussure. Para aquéllos lo real, lo científico, lo objetivo, era la lengua en su devenir, en su realidad cambiante e histórica, mientras que el estado de lengua ', el momento fortuito y aislado de una evolución, es sólo una abstracción operada por el investigador. Saussure, sin embargo, señala que el carácter abstracto sólo es atribuible mediante el criterio de la conciencia del hablante 2 (Engler, 1988: 133), para quien sólo tiene existencia real el estado de lengua (estado sincrónico) en el que vive, mientras que su historia, en el hablante medio, suele carecer de toda presencia ${ }^{3}$. Así, la teoría saussureana supone una inversión de los términos, pues se considera que

1 «La nazione di stato di lingua risale almeno agli ultimi decenni del secolo scorso; però non è stata veramente chiarita finchè non sia stata ricondotta a quella di sistema o struttura)" (Godel, 1984: 177).

2 Strozier, en un elegante artículo, ha recordado que la tradición intelectual de Saussure le ancla en las concepciones mentalistas del pasado siglo; aspecto éste que no ha sido apenas recogido por la tradición saussureana, dice, hasta Benveniste o Derrida (1985: 34 ss).

${ }^{3}$ Tal reclamo a la conciencia, o competencia del sujeto es recurrente en el Cours de Linguistique Générale. «Eppure la necessità di considerare le lingue anche, o anzitutto, dal punto di vista sincronico, prescindendo cioè dal passato, appare non appena ci si pone nella situazione del soggetto parlante. La gente che parla e capisce una lingua ignora del tutto i fatti storici che l'hanno fatta tale qual è: questi non entrano nell'acquisizione della sua "competenza") (Godel, 1984: 171). 
sólo lo sincrónico tiene una existencia concreta real, mientras que los aspectos evolutivos, accesibles sólo al investigador, ofrecen un elevado grado de abstracción. $\mathrm{O}$, con mayor precisión, se opera una doble abstracción, sobre ambos ejes:

Pour Saussure, le linguiste opère dans ce continuum historique qu'est diachronique, double abstraction de dégagement des rapports systématiques sur l'axe des simultanéités ou de reconstruction sur l'axe des succesivités (Buridant, 1984: 20) ${ }^{4}$.

En segundo lugar, la dicotomía crónica opera sólo sobre la lingüística, y no sobre la lengua, como se han encargado de subrayar Coseriu (1958: 26ss.r) y Godel (1984: 169). Es importante destacar que la lengua no reacciona ante las posibles divisiones de la lingüística, y que método y objeto no se pueden confundir. Metodológicamente, el pensamiento del Saussure del CLG es muy deudor del de la Mémoire: a pesar de la importancia y privilegio del análisis sincrónico, la perspectiva diacrónica no se puede obviar (CLG, I.III, passim). Esto era ya algo asumido desde la evidencia de la irregularidad sincrónica que manejaban los neogramáticos (cf. Bynon, 1977: 35ss.t): la existencia en cada estado sincrónico de elementos no asimilables a las leyes sincrónicas apela a la existencia de parámetros diacrónicos que pueden alcanzar el estatuto de leyes diacrónicas.

Rosen (1986) se ha preocupado de señalar el distinto funcionamiento de las leyes sincrónicas y diacrónicas en el CLG: según él, lo que Saussure pretendía al plantear su existencia no era tanto distinguir entre sincronía y diacronía cuanto profundizar en el concepto de ley lingüística (CLG, I.III.6). Su objetivo sería señalar que tal concepto ofrece la complicación de no funcionar igual en ambas perspectivas, ya que la ley diacrónica (reducida en tiempos de Saussure a ley fonética) se impone a la lengua, pero no es general, ya que escasamente afecta a la morfosintaxis, mientras que la ley sincrónica es general pero no es imperativa, sino que es la pura constatación de un dato.

La paradoja de la trayectoria intelectual de Saussure es evidente: lingüista histórico durante toda su vida, llega a proponer al final una lingüística sincrónica radicalmente diferente. Los sucesivos comentaristas del CLG han subrayado siempre su gran innovación, la sincronía, pero ya Godel, entre otros, ha demostrado que para Saussure la diacronía debería ser la introducción previa a la sincronía. Buyssens ha dedicado un artículo

\footnotetext{
${ }^{4}$ No se puede dudar sobre el carácter abstracto de toda operación basada en la lengua: «La science des évolutions de la langue, comme celle des états de langue, ne saisit son objet que par approximation et ne cannaît que des vérités générales qui si dégagent des faits et en sont une image simplifiée. Seule la linguistique de la parole organisée garde le contact avec la réalité [...]» (Sechehaye, 1940: 30).
} 
precisamente a mostrar que la formulación sincronicista de Saussure no logra despegarse totalmente de la dimensión evolutiva ${ }^{5}$. La noción de sistema que encontramos en el CLG procede de una genial concepción presente ya en la Mémoire, deudora en principio de Grimm: la de que todo sistema se mantiene intacto en su evolución, porque las modificaciones atañen sólo a sus elementos. El sistema permanece a través de sus modificaciones, de la misma manera que cualquier modificación es inconcebible fuera del sistema. De esta manera Saussure había logrado en la Mémoire describir los sistemas fonológicos indoeuropeos sin necesidad de atribuir un contenido positivo a cada fonema; y de ahí surge la definición del sistema no como suma jerarquizada de elementos, sino como relación de diferencias:

La langue est un système dont tous les éléments sont solidaires et où la valeur de l'un ne résulte que de la présence simultanée des autres. Dans la langue il n'y a que des différences sans termes positifs (CLG, II.IV.2; 4).

Los conceptos de identidad y diferencia llegan a constituir a partir de esta concepción los verdaderos móviles de todo un sistema de pensamiento. Piénsese que toda la cuestión del valor dentro del sistema, que tanto preocupó en los estructuralismos, se funda precisamente en el juego de identidades y, más aún, de diferencias, que evita los problemas originados por la continua modificación de su contenido positivo ${ }^{6}$. Pero sorprende que llegado a este punto Saussure no vuelva sobre la diacronía para intentar configurar la noción de sistema diacrónico, toda vez que ya disponía de leyes y elementos sin duda bien conocidos; porque el sistema no permite ver lo histórico: en realidad la noción de sistema se presenta como sustituto de la mucho más evanescente ${ }^{7}$ de estado de lengua (cf. Buyssens, 1961: 28).

Buridant (1984) y Engler (1988) han recordado el equilibrio metodológico que en el CLG se sostiene entre estudio sincrónico y diacrónico para denunciar las tergiversaciones a que fue sometido con posterioridad. A pesar del debate sobre la precedencia lógica entre sincronía y diacronía, llegó a constituir un lugar común entre muchos estructuralismos la idea de

\footnotetext{
${ }^{5}$ Por lo tanto no dejan de parecer excesivamente simplificadoras afirmaciones muy generalizadas como que «sólo el concepto de sincronía le importaba a Saussure, pues le permitía fundar la lingüística como estudio de sistemas coherentes» (Greimas-Courtés, 1979: 120).

" "Thus a given concept can be defined more clearly by its place in a given conceptual structure than by definition of its own content, for the latter is subject to constant change and reinterpretation", dice Winner (1976: 6-7r) refiriéndose a la obra de Mukarovsky. Para una revisión de las críticas de los praguenses a la diferencia saussureana, en especial las de Karcevski, vid. Percival (1981: 41, passim.).

7 Aunque en esto Bally no esté de acuerdo: «Chez Saussure, donc, la notion d'état est une fiction méthodologique du linguiste, ce qui le différencie de Bally pour qui la notion d'état est une réalité subjective qui permet le fonctionnement de la parole» (Buridant, 1984: 23).
} 
que el único estudio válido era el sincrónico. Lo cual suponía olvidar el espíritu historicista del maestro ginebrino ${ }^{8}$ para centrarse exclusivamente en sus grandes innovaciones frente a los neogramáticos, es decir, el aparato sincrónico. En esto se evidencia también el clima cultural de comienzos de siglo, dominado por el formalismo y el anti-historicismo. Pero asimismo subyace la tergiversación más arriba mencionada, contra la que reacciona Coseriu, según la cual se pretende atribuir a Saussure una identificación entre lengua y sincronia, a pesar de que en todo el CLG es evidente la caracterización puramente metodológica de la sincronía, que jamás se cruza con la realidad objetual de la lengua ${ }^{9}$.

Además de esta tergiversación de la dicotomía crónica que en su tiempo afectó a los nacientes estructuralismos de manera notable, se ha hablado también de una cierta mitificación. En un polémico artículo, Percival (1981) intenta mostrar que la influencia de Saussure sobre las escuelas de Praga y Copenhague fue mucho más escasa de lo que habitualmente se ha reconocido. Aunque tal aportación nos parece un tanto superflua (la obra ya está hecha y superada), nos interesa aclarar un aspecto: concluye Percival que «the Cours was not greeted on its appearance as a revolutionary work» (1981: 43), basándose en que la oposición sincronía-diacronía se encuentra ya en Comte y Jespersen, y que además tergiversa esa ya conocida oposición. Concluiremos a nuestra vez recordando que la labor de Saussure no fue, en efecto, inventar la oposición, que por cierto es bastante más antigua que las obras de Comte y Jespersen ${ }^{10}$, sino profundizar en los métodos que cada perspectiva exige, empezando por crear un nombre neológico ${ }^{11}$, imponiendo el nivel metodológico al nivel factual ${ }^{12}$, y culminando con una

8 «Plus on étudie la langue, plus on arrive à se pénétrer de ce fait que tout dans la langue est histoire, c'est-à-dire qu'elle est un objet d'analyse historique, et non d'analyse abstraite, qu'elle se compose de faits et non de lois, que tout ce qui semble organique dans le langage est en réalité contingent et complètement accidentel» (Saussure, 1967: 3283.15).

9 Buridant denuncia el uso de pasajes certainement réducteurs del CLG por parte de W. von Wartburg con el objeto de achacar al ginebrino la irreductibilidad de las dos lingüísticas. E insiste de nuevo sobre la esencial unidad del objeto defendida por Saussure (cf. Buridant, 1984: $25 n$ ). En un sentido mucho más amplio, también Strozier ha hablado de una tergiversación generalizada del pensamiento saussureano, pero «it is a misreading that any age makes of the previous one and that allows later theorists access to their predecessors [...] Such misreadings constitute the history of theorym (1985: 47).

${ }_{10}$ Se han señalado, entre otros: James Harris, Hermes, or a Philosophical Inquiry Concerning Language and Universal Grammar (London, 1751; trad. fr., 1796), que opone punto de vista etimológico y punto de vista sistemático; el famoso prólogo de la Gramática de Bello (1847); Georg von der Gabelentz, Die Sprachwissenschaft (Leipzig, 1891), de influencia decisiva sobre Saussure (aunque sobre esto convendría revisar con calma ciertas aportaciones de Koerner; cf. 1988: 53 ss); O. Dittrich, Grundzüge der Sprachpsychologie (Halle, 1903), quien distingue entre synchronistic y metachronistic; T.G. Masaryk, Ensayo de una lógica concreta (1885), quien distingue de manera sistemática la lingüistica histórica de la estática. Su impronta hegeliana, en fin, se localiza en la herencia de Victor Henry.

11 "Les termes "diachronique" et "diachronie" ont été créés par Saussure; il n' y pas a en d'antécédents à ma connaissance» (Koerner, 1980: 100).

12 «Par une décision théorique un ordre de phénomènes a été isolé et opposé à un autre; une méthode propre est assignée à chacun des ordres et deux disciplines indépendents sont 
fundamental modificación del concepto histórico de la lengua: si durante el comparatismo se entendía como la búsqueda de una perfección de los orígenes, y los neogramáticos buscaban las leyes evolutivas según la metáfora biológica (Engler, 1988), Saussure logrará definir la diacronía como sucesión de estados sincrónicos. Tal concepción logra una ecuación fundamental en la unidad de la lingüística: todo estado remite a una evolución, toda evolución remite a la idea de estado.

La formación de la perspectiva diacrónica parte, pues, de las concepciones tradicionales de la gramática histórica, pero aporta la idea fundamental, no desarrollada por Saussure, de que si el cambio se opera sobre el sistema sin que éste se modifique, el cambio tiene necesariamente que responder a un principio regularizador ${ }^{13}$. Éste será el inicio de la diacronía estructural.

2. Estructuralismo diacrónico. El desarrollo teórico que la diacronía saussureana conoció en la escuela de Praga, tanto en la vertiente lingüística como en la literaria, se centró especialmente en la reivindicación de su carácter sistemático y en el debilitamiento de la fuerte antinomia con la sincronía ${ }^{14}$. La obra de Saussure se había detenido en la construcción de una lingüística sincrónica, caracterizada como la única susceptible de reconocer sistemáticamente sus elementos, frente a la neogramática de, por ejemplo, Paul (1886), para quien sólo lo histórico es científico ${ }^{15}$. La primera operación de lo que luego sería el Círculo de Praga es constatar que también lo diacrónico ha de ser sistemático. Se ha atribuido (Galán, 1984: 25t: Koerner, 1988: 46) a Saussure una sobre-reacción sincronicista ante la filología neogramática, que le habría llevado no sólo al establecimiento de la fecunda pareja conceptual, sino también a una comprensión del paradigma diacrónico excesivamente mediatizado por el atomismo filológico que tan bien había conocido en la época de la Mémoire; pero dentro de la propia escuela ginebrina Sechehaye intentaría una primera relativización de la oposición, que, a pesar de su ingeniosa utilización de lo pregramatical como motor del cambio lingüístico, descuida en exceso la diferencia entre langue y parole (Sechehaye, 1940: 7-8; Engler, 1988).

constituées» (Engler, 1988: 127-128). La brillantísima y bien documentada aportación de Engler al coloquio La diachronie hier et demain (Lille, 21-23 de octubre, 1982) cifra precisamente en la nueva dimensión diacrónica una de las grandes aportaciones del maestro ginebrino.

${ }^{13}$ Buyssens, en todo caso, recuerda que Saussure nunca llega a localizar en el sistema el origen del cambio (1981: 152).

${ }_{14}$ Los intentos de colmar la laguna entre sincronía y diacronía proceden ya de la propia escuela de Ginebra: Sechehaye consideraba una hipotética linguiística del habla como decisiva en este sentido (cf. Godel, 1984: 183).

15 «Although [...] Paul gave full credit only to historical linguistics he admitted the existence of a "deskriptive Grammatik" whose heuristic value he acknowledged. Saussure tended, perhaps as the result of an overreaction against Paul's claim, to reverse the picture emphasizing the predominance of the "fait synchronique" over the evolutionary aspect of language. A number of correspondences, however, can be detected in connection with the opposition of these two methods of linguistic investigation [...]» (Koerner, 1988: 46). 
El fundamental manifiesto de Tynianov y Jakobson, Problemas en el estudio de la literatura y el lenguaje (1928), supone la introducción de la noción de sistema en el paradigma diacrónico, afectado por los avances logrados en la sincronía ${ }^{16}$. Ambas perspectivas se ven consiguientemente hermanadas mediante el punto de vista funcional:

La historia del sistema es a su vez un sistema. El sincronismo puro se presenta ahora como una ilusión: cada sistema sincrónico contiene su pasado y su porvenir como elementos estructurales inseparables del sistema (1928: 104t).

Sin embargo, la interpretación asistémica de la diacronía, basada en la idea saussureana del carácter fortuito del cambio, perduró incluso a nivel teórico durante bastante tiempo ${ }^{17}$, lo cual produce una enorme extrañeza si consideramos que este breve pero rotundo manifiesto es la pieza clave para comprender el paso del formalismo de OPOJAZ (recuérdese El problema de la evolución literaria de Tynianov) al estructuralismo de Praga, cuyas famosas tesis llegarán sólo un año después (1929). La incomodidad de Jakobson ante la diacronía saussureana era ya patente de antes, y su aportación clave en estos años consistirá en deshacer las ecuaciones sincrónico $=$ estático y diacrónico $=$ dinámico, deducibles del pensamiento de Saussure ${ }^{18}$.

Desde las tesis de 1929 ya no se puede entender la sincronía como mera descripción estática de sistemas, ni la diacronía como dinamismo evolutivo. La noción jakobsoniana de sincronía dinámica se funda sobre la realidad de que en todo sistema existen puntos de roce, desequilibrios y junturas, que no pueden escapar al análisis sincrónico; son, por ejemplo, los denominados puntos débiles del sistema, entendidos como insuficiencias del instrumento lingüístico ante necesidades expresivas no previstas (cf. Coseriu, 1958: 197r). La diferencia fundamental entre sincronía dinámica

16 «Un vasto programa de estudio de carácter funcional, propuesto por los dos destacados teóricos de la lengua y las formas literarias, vendría a salvar en su opinión las deficiencias de ambas perspectivas aisladas. La determinación horizontal, sincrónica, de las formas literarias, precedida por la descripción funcional de sus elementos integrantes, sólo adquiere plenitud de sentido al rebasar el ámbito restringido de la serie literaria; y, por tanto, la descripción diacrónica, en función de la estructura sincrónica de la serie literaria [...] no puede tampoco prescindir, a ninguno de sus niveles, de establecer el enlace funcional con las demás series culturales») (García Berrio, 1973: 294).

17 «Hasta la fecha, la mayoría de las especulaciones estructuralistas francesas están atrapadas en los confines del análisis sincrónico. En la proclamación de Jakobson y Tynjanov presenciamos la primera integración de las dos dimensiones, vistas como complementos dinámicos» (Galan, 1984: 25t).

${ }_{198}$ Cf. Jakobson, 1963: 36ss, 1970: 460. En palabras de Garrido Gallardo: «No se debe confundir, pues, sincronía y estática: en cada fase hay formas más conservadoras y formas más innovadoras que reclaman respectivamente la atención sobre los factores permanentes a la vez que el enfoque histórico de los factores de cambio» (1987: 19). 
y diacronía estructural sigue presente en el hecho de que la primera estudia las tensiones dentro del sistema mientras que la segunda exige la confrontación de al menos dos sistemas discretos. La asunción de una sistemática del cambio, unida a la posibilidad de un enfoque dinámico del estado de lengua, niega la infranqueabilidad de la frontera metodológica entre ambas perspectivas.

En 1934 Hjelmslev se había situado ya en la perspectiva diacrónica estructural, admitiendo la sistematicidad de algunos cambios lingüisticos, pero preocupado todavía más por sus causas que por sus efectos. En su opinión, los cambios que afectan al sistema lingüístico tienen su origen en el sistema mismo, y se dirigen a completar un estado óptimo exigido por el propio sistema (1934: 228-229t). Lo cual viene a subrayar el hecho de que no todas las modificaciones sufridas por el lenguaje son sistemáticas ni afectan al sistema ${ }^{19}$ : las modificaciones de contenido y de estructura o realización externa (1934: $57 \mathrm{t}$ ) están en todo momento supeditadas a las posibilidades del sistema y dificilmente le interfieren:

El sistema, la forma, domina sobre la estructura y el contenido y los obliga a no experimentar ningún cambio que vaya contra la finalidad del sistema (1934: 62t).

Más desafortunada parece la alusión a un estado óptimo para cada sistema, en la que resuenan ecos del teleologismo de Praga. Entre causalidad y teleologismo en el cambio lingüístico se ha dado a menudo una confusión peligrosa, sobre todo con la noción de tendencia de las lenguas, de raigambre positivista y evolucionista. Tal tendencia interna del sistema, o finalidad intrínseca, es muy dificil de mantener; como dice Coseriu, la lengua como hecho objetivo, como técnica histórica del hablar, no tiende $y$ no puede tender a nada ${ }^{20}$. Lo que Hjelmslev parece estar adelantando con su idea de estado óptimo es precisamente lo contrario: no la tendencia hacia un estado ideal, sino la debilidad actual que mediante el cambio se pretende corregir. Lo que en teoría de sistemas se denominará retroalimentación.

Es importante subrayar el hecho de que el desarrollo de la diacronía no ha avanzado gran cosa desde las formulaciones de la escuela de Praga.

19 «Las demás modificaciones, es decir las modificaciones simplemente en la norma o en el uso, no concuerdan con el sistema, tienen una cierta relación con el sistema en la medida en que el sistema en cualquier momento las mantiene en jaque y les impide ir más allá de lo que el sistema permite. Pero no están ocasionadas por el sistema y no tienen ninguna influencia sobre el mismo» (1934: 64t).

20 «En general, las afirmaciones teleológicas no son explicaciones y carecen de valor cognoscitivo, pues la "finalidad objetiva" no es algo comprobable [...] sino que sólo manifiesta una actitud del sujeton (1958: 227-228r). El propio Saussure subraya a menudo que la lengua no premedita nada (cf CLG, I.III). El sistemismo abrirá otras perspectivas. 
El paradigma diacrónico se ha visto conformado como la búsqueda de las leyes que rigen el cambio lingüístico, sometido así a una estructura funcional. El teleologismo del que a menudo se ha hablado para referirse a esta acepción del cambio reside en una incidencia especial sobre la noción de cambio frente a la de evolución. Se busca el resultado dentro del sistema, y no en los intersticios entre sistemas, de manera que lo que se resalta es la incidencia de la innovación sobre la totalidad del sistema al que afecta, y no su génesis.

El sistema, concebido como un lugar où tout se tient, es capaz de albergar las modificaciones de sus elementos mediante mecanismos autorreguladores, porque, volviendo a Saussure, su principio fundamental es la diferencia entre términos negativos: toda innovación obliga a una reestructuración de las relaciones entre los elementos, y no necesariamente a un cambio de elementos. Desde este punto de vista ya no es preciso acudir a la génesis del cambio para lograr una explicación, sino que su importancia radicará en sus efectos sobre el sistema. Esto, por una parte, supone la imbricación definitiva de sincronía y diacronía (todo cambio observable opera sobre un sistema, y todo sistema es objeto de cambio); pero por otra parte confiere al teleologismo jakobsoniano una dimensión funcional, interna a la noción sistémica, y no una especie de determinismo lingüístico, de dificil justificación.

Los criterios de rendimiento funcional y de economía de Martinet insertan el problema del cambio en la órbita estructural. El principio de economía opera con la antinomia de las necesidades comunicativas frente a la tendencia humana al mínimo esfuerzo:

Une expansion non-économique est une expansion qui entraînerait une dépense d'effort plus grande que celle que la communauté juge en valoir la peine dans une situation donnée. Une telle expansion sera stoppée. L'inertie, lorsqu'on la sent excessive, c'est-à-dire nuisible aux intérêts légitimes de la communauté, sera sévèrement réprimée (Martinet, 1955: 94).

El principio de economía, versión funcional de la ley del mínimo esfuerzo (Zipf, 1949), puede explicar la aparición de innovaciones consentidas por el sistema, la desaparición de entidades funcionalmente innecesarias o el refuerzo de lo necesario, siempre en consonancia con las necesidades expresivas. Por eso Coseriu prefiere entenderlo como principio de eficiencia técnica o de necesidad expresiva (1958: 202-204r). Todo cambio en el sistema, ya sea relativo al mismo o condicionado por factores extralingüísticos, vendrá regulado por este principio de tensión que, sin prever la evolución concreta de cada momento, es capaz de localizar un buen número de evoluciones improbables. Lo que consigue una teoría funcional como la de Martinet (y en este sentido se puede considerar más operativa que la de Hjelmslev) es interrelacionar los aspectos sistemáticos con las necesidades 
comunicativas, lo que es casi equivalente a conjugar langue y parole, dando así un paso en la dirección que proponía Sechehaye para superar las limitaciones de una lingüística exclusivamente sincrónica de la langue.

3. El acronismo. Frente al floreciente desarrollo del paradigma diacrónico observado por la escuela de Praga, las ulteriores reelaboraciones de la herencia saussureana se dirigen con absoluta prioridad bien al asentamiento exclusivo de la sincronía, bien a una superación de la dicotomía crónica. La trayectoria de Hjelmslev, por ejemplo, muestra cómo el espíritu de la escuela de Copenhague se sitúa en la línea de quienes pretendieron llevar la dicotomía saussureana a su máxima polarización, atribuyendo carácter científico sólo a la perspectiva sincrónica. Afirmaciones de 1928 como que «el sistema es eminentemente sincrónico o que la gramática es por definición sincrónica, prestándose sólo secundariamente al estudio diacrónico, que es extraño a la naturaleza misma del sistema gramatical en cuanto tal» $(1976: 57,62)$ son ortodoxamente saussureanas, pues por una parte identifican sincronía y sistema y por otra no olvidan la importancia, eso sí, secundaria, de la diacronía. Sin embargo la obra diacronizante de los praguenses no podía pasar inadvertida ni siquiera para una lingüística tan logicista como la de Hjelmslev, y de hecho en 1950 reconocería en Upsala la sistematicidad de la diacronía ${ }^{21}$. La identificación de sistema y sincronía parecería entonces haber perdido su función dentro de la economía de la epistemología lingüística, ya que si el cambio, procedente de la parole, también era susceptible de sistematización a través de sus efectos en la langue, la diacronía podría considerarse tan sistemática como la gramática hjelmsleviana.

Greimas nos proporciona una clave de la solución adoptada por los lingüistas daneses, que sirvió tanto para la formación de la glosemática como para el esclarecimiento de las tesis semióticas del grupo de París. Muchas aplicaciones muy posteriores de los métodos semióticos parecen retrotraerse a la época en que Hjelmslev todavía no reconocía la sistemacidad de la diacronía, probablemente ignorando que ya en $D u$ sens, Greimas había postulado la superación de la dicotomía crónica a través de una concepción acrónica de la investigación lingüística: la significación en sí es intemporal e inubicua, sólo los medios de su manifestación, el plano de la expresión, es temporal $(1970: 116 \mathrm{t})^{22}$. Lo

${ }^{21}$ Muraro, 1971. Bertil Malmberg ha meditado sobre ese artículo: «Si, dans sa conférence de 1950, Hjelmslev a trouvé bon d'attirer l'attention de son public au caractère systématique des chagements et à l'aspect structural de l'évolution, ç'a dû en partie être parce que, malgré Jakobson et les Pragois, l'application du structuralisme dans la diachronie était nouvelle à l'époque et méritait l'attention» (1976: 198).

${ }^{22}$ En los estudios literarios se llega a una conclusión similar; tras las largas polémicas entre historia y crítica, que se remontan a las obras de Menéndez Pelayo, Brunetière o Croce y que alcanzan su apogeo en la famosa diatriba entre Barthes y Picard, se alcanzan con la semiótica propuestas claramente tendentes al acronismo crítico (cf. Moisan, 1987). 
que subyace, entonces, es el descrédito en que ha caído la sincronía, desde el momento en que, aunque permite reflexionar sobre el sistema, no es útil para analizarlo ${ }^{23}$. La tesis greimasiana parte de considerar que los dos conceptos saussureanos se sitúan en los polos de un eje de la temporalidad, que él denomina el eje de la cronía (1970: 118t), opuesto lógicamente a la dimensión acrónica:

La acronía sirve para afirmar el carácter atemporal de las estructuras lógico-matemáticas, al tiempo que la no pertinencia de la dicotomía saussureana [...] Desde el punto de vista de la teoría semiótica, cabe considerar a las estructuras semióticas profundas como acrónicas, mientras que las estructuras discursivas -más superficiales - precisan de la temporalización (Greimas-Courtés, 1979: 22-23t).

Es evidente que la concepción hjelmsleviana de la lingüística descansa ya no sobre una base sincronicista, ligada al eje crónico, sino sobre un acronismo de raigambre lógico-formal como el que describe Greimas. Pero es preciso observar que una vez más la tensión entre sincronía y diacronía queda sin resolver, ya que esta superación es más bien un soterramiento: al pasar de la dicotomía sincronía-diacronía a la oposición crónico-acrónico se opera un cambio de nivel epistemológico, de la lingüística a la epistemología. Recordemos que ya Coseriu (1958) había denunciado una confusión similar a la hora de diferenciar entre lenguaje y lingüística (sincronía y diacronía eran perspectivas lingüísticas, no hechos de lenguaje); ahora, el paso propuesto asciende de la lingüística como ciencia del lenguaje a la epistemología como ciencia de la metodología. La dialéctica crónico-acrónico no es lingüística como lo es la de sincronía-diacronía, sino de un nivel superior, aplicable tanto a la ciencia lingüística como a cualquier otra. En otras palabras, la propuesta greimasiana, que explica el posicionamiento de Hjelmslev y de toda la lógica semiótica, prescinde explícitamente de toda la polémica entre estado y devenir, ya que considera ambas instancias como pertenecientes a una misma sistemática de lo temporal, ajena a las pretensiones atemporales de una lógica de sistemas cerrados ${ }^{24}$.

Lo que podríamos denominar como resistencia atemporal (las gramáticas lógico-formales, incluida la formulación semiótica de los años 50-60) se caracteriza efectivamente por adoptar una lógica de sistemas cerrados,

23 «Efectivamente, la noción de sincronía es tan imprecisa como, por ejemplo, la de presente [...] Un estado de lengua - por lo tanto, una sincronía - dura varios cientos de años y comprende transformaciones internas [...] numerosas y variadas» (Greimas-Courtés, 1979: 380t).

${ }^{24}$ La definición del sistema semiótico a través de su coherencia lógica interna antes que por la sincronización de sus elementos, por una parte, y la equiparación de la distancia entre dos estados de lengua al comparatismo de lenguas coexistentes, por otra, muestran hasta qué punto la perspectiva acrónica se impone a la pareja sincronía-diacronía. 
es decir, por considerar que su objeto tiene una estructura sistemática aislada del entorno. Ricoeur lo ha expresado así:

La lingüística estructural procede de una decisión de carácter epistemológico, la de mantenerse en el interior de la clausura del universo de los signos; en virtud de esta decisión, el sistema no tiene afuera; es una entidad autónoma de dependencias internas (1969: 95t).

La existencia de tales sistemas cerrados, sin embargo, es ficticia (Ashby, 1962); en el mejor de los casos responde a meras hipótesis de trabajo exigidas por la investigación. La teoría de sistemas, desde von Bertalanffy, ha insistido a menudo en que la principal característica de todo sistema reside precisamente en su equilibrio constante, garantizado por los mecanismos de autorregulación que aseguran la entrada y salida de elementos:

Los únicos cambios que pueden afectar a la propia organización [...] deben producirse fuera del sistema. Pero esto es posible de dos modos distintos: o un programa preciso, inyectado en el sistema por un programador, determina los cambios [...]; o éstos son determinados todavía desde el exterior, pero por factores aleatorios [...] (Atlan, 1979: 47t).

Respondiendo aproximadamente al primer caso, la famosa ley de Grimm, tan rentabilizada por Saussure, encuentra en la formulación del 'sistemismo una confirmación definitiva: el sistema está equilibrado en todo momento. Pero ello se debe no a que el sistema esté aislado del entorno (las ideas de entorno y sistema son indisolubles), sino a que la dinámica entre sistema y entorno está perfectamente regulada por el sistema mismo ${ }^{25}$.

Esto no quiere decir que el acronismo lingüístico sea falaz. De ser así, toda la perspectiva sincrónica clásica, que reducía la lengua al estado de lengua, incluso a la langue estática y abstracta, sería también un error. Pero hay que distinguir entre sistema y punto de vista. Lo susceptible de ser enfocado sistémicamente es el objeto, el lenguaje; el punto de vista, sincrónico, diacrónico o acrónico; puede ser sistémico o asistémico, no es lógicamente necesaria ninguna alternativa. Tanto la glosemática como la

25 «Esta característica de los sistemas de manifestarse mediante configuraciones diferenciadas que se corresponden de manera regular con ciertos elementos de su entorno es lo que llamamos adaptación o adecuación del sistema [...] Comprender un sistema supone necesariamente comprender el tipo de entornos para los que resulta adaptado y las condiciones que le permiten adaptarse a ellos" (Teso, 1988: 45-46). Von Bertalanffy liga con la vieja cuestión del teleologismo mediante el criterio de adaptabilidad: el comportamiento teleológico, dice, «es una forma de comportamiento definible en términos científicos y cuyas condiciones necesarias y mecanismos posibles pueden ser indicados» (1968: 47). 
semiótica han atribuido siempre a la lengua el carácter de sistema abierto; pero, sobre todo la primera, han tendido a analizarlo con una perspectiva parcial, centrándose en los aspectos lógicos y relegando toda la faceta de sus relaciones con el entorno: usos individuales, evolución, interferencias con otros sistemas, etc. El desarrollo de la pragmática sirvió a la semiótica para corregir este desenfoque inicial, y su desarrollo supuso, como sabemos, el replanteamiento general de toda la disciplina; pero curiosamente en los orígenes de esa apertura se encuentra una apelación casi sechehayana a los valores del habla, ya que si en un primer momento se habían opuesto lo sistemático y lo histórico, lo evenemencial y lo virtual, la elección y la obligación, la innovación y la institución, en un segundo momento se hace precisa una síntesis, localizable sólo en el discurso, en el acto del decir ${ }^{26}$.

En esta evidente limitación se encuentra la explicación de por qué desde Jakobson y la escuela de Praga se ha atenuado tan drásticamente la investigación acerca del cambio lingüístico. Una prueba de ello es que la teoría de sistemas, centrada en la noción de sistema abierto, sí ofrece desarrollos sobre la idea de novedad, mientras que ni la lingüística formal ni la semiótica parisina ofrecen nuevas soluciones. En este sentido resulta curioso comprobar que la teoría de sistemas avanza en la línea estructural propuesta, entre otros, por Jakobson cuando hablaba de sincronía dinámica como estudio de los efectos del cambio dentro del sistema, presente en muchas concepciones de Martinet.

Deberíamos concluir, siguiendo a Pomian, que las fases del estructuralismo que pretendieron cerrar epistemológicamente sus sistemas de estudio renunciaron no sólo a la dimensión histórica, sino, lo que es más grave, a la idea de tiempo. Frente a estructuralismos más arriesgados, como el ya mencionado de Jakobson, o la morfogénesis estructural de Thom, sustrajeron de la noción clave de estructura toda implicación dinámico-temporal 27 ; el mismo Eco reconoce que toda la polémica entre estructura e historia ha sido posible porque desde un principio la primera fue tomada como negación de la segunda, afirmando la superioridad de lo idéntico sobre lo cambiante ${ }^{28}$.

${ }^{26}$ «El surgimiento del decir en nuestro hablar es el misterio mismo del lenguaje; el decir, es esa que yo llamo la abertura, o mejor, la apertura del lenguaje» (Ricoeur, 1969: 108t).

${ }_{27}$ "Car on ne peut tenir pour entièrement satisfaisantes les équivalences établies dans cet article entre "structure" et "ensemble de relations pensées comme logiques, rationnelles et telles qu'on puisse déduire ou prévoir à l'avance les transformations de l'ensemble connaissant le chagement d'une de ses composantes" ou autres propositions du même genre" (Pomian, 1984: $211)$.

${ }_{28}$ «Si se ha podido orquestar una batalla tan encarnizada entre estructura e historia ha sido porque la estructura en cuestión no era considerada como un medio de investigación sincrónica de unos fenómenos afectados por una historicidad total, sino porque ya desde su inicio se la consideraba como negación de la historia, en cuanto aspiraba a ser el fundamento de lo idéntico» (Eco, 1968: 393t). 


\section{Referencias bibliográficas}

ASHBY, W. R. (1962): «Principles of the Self Organizing System». En Principles of Self Organization, VV.AA, 255-278, Pergamon.

AtLAN, Henri (1979): Entre le cristal et la fumée, Paris: Seuil; trad. cast. (1990). Entre el cristal y el humo. Ensayo sobre la organización de lo vivo, Madrid: Debate.

BerTalanfFy, Ludwig von (1968): General System Theory. Foundations, Development, Applications. New York: Braziller. Trad. esp. (1976). Teoría general de los sistemas. Fundamentos, desarrollo, aplicaciones. México: FCE.

BURIDANT, Claude (1984): "Saussure et la diachronie». Travaux de Linguistique et de Littérature 22/1, 19-51.

BUYSSENS, Eric (1961): «Origine de la linguistique synchronique de Saussure». Cahiers Ferdinand de Saussure 18, 17-33.

BYNON, Theodora (1977): Historical Linguistics. Cambridge University Press. Trad. esp. (1981) Madrid: Gredos.

COSERIU, Eugenio (1958): Sincronía, diacronia e historia. El problema del cambio lingüistico. Montevideo. Reimpr. (1973) Madrid: Gredos.

ECO, Umberto (1968): La struttura assente. Milano: Bompiani. Trad. esp. (1989) La estructura ausente. Introducción a la semiótica. Barcelona: Lumen.

ENGLER, Rudolf (1988): «Diachronie: l'apport de Genève». Cahiers Ferdinand de Saussure 42, 127-166.

Galan, Frantisek W. (1984): Historic Structures: The Prague School Project,19281946. University of Texas Press. Trad. esp. (1988) México: Siglo XXI.

GARCÍA BERRIO, Antonio (1973): Significado actual del formalismo ruso. (La doctrina de la escuela del método formal ante la poética y la lingüística modernas). Barcelona: Planeta.

Garrido Gallardo, Miguel Angel (1987): «Jakobson y la semiótica literaria». En La crisis de la literariedad, VV.AA, 11-25, Madrid: Taurus.

GODEL, Robert (1984): «Sincronia, diacronia e pseudo-diacronia». Cahiers Ferdinand de Saussure 38, 169-187.

GreIMAS, Algirdas Julien (1970): Du sens. Essais sémiotiques. Paris: Seuil. Trad. esp. (1973) En torno al sentido. Ensayos semióticos. Madrid: Fragua.

Greimas, A. J.-Courtés, J. (1979): Sémiotique. Dictionnaire raisonné de la théorie du langage. Paris: Hachette. Trad. esp. (1982) Madrid: Gredos.

HJELMSLEV, Louis (1934): Sistema lingüístico y cambio lingüístico. Madrid: Gredos.

HJelmslev, Louis (1976): Principios de gramática general. Madrid: Gredos.

JAKOBSON, Roman (1963): Essais dè linguistique générale. Paris: Mouton.

- (1970): Main Trends in the Social and Human Sciences, I. Mouton \& Unesco.

KOERNER, Konrad (1980): «Sur l'origine du concept et du terme de 'synchronique'en linguistique». En Recherches de linguistique, 100-109, Bruxelles: Ed. de l'Université.

- (1988): Saussurean Studies / Etudes saussuriennes. Genève: Slatkine.

LLOVET, Jordi (1977): «Jan Mukarovsky, un signo nuevo para la estética». En Escritos de Estética y Semiótica del Arte, VV.AA, 9-29, Barcelona: G. Gili.

MALMBERG, Bertil (1976): «Langue-forme-valeur: réflexions sur trois concepts saussuriens». Semiotica 18, 195-200. 
MARTINET, André (1955): Economie des changements phonétiques. Traité de phonologie diachronique. Berne: Francke.

MoISAN, Clément (1987): Qu'est-ce que l'histoire littéraire? Paris: PUF.

Muraro, Luisa (1971): «Hjelmslev lettore del Corso di Linguistica Generale». Cahiers Ferdinand de Saussure XXVII, 43-53.

Paul, H. (1886): Prinzipien der Sprachgeschichte. Halle.

Percival, Walter Keith (1981): «The Saussurean Paradigm: Fact or Fantasy?» Semiotica 36, 33-49.

POMIAN, Krzysztof (1984): L'ordre du temps. Paris: Gallimard.

RICOEUR, Paul (1969): Le conflit des interprétations. Paris: Seuil. Trad. esp. parcial (1975) en Hermenéutica y estructuralismo, Buenos Aires: La Aurora.

Rosen, Haiim B. (1986): «Les lois synchroniques et les lois diachroniques dans le Cours de Saussure». Cahiers Ferdinand de Saussure 40, 91-103.

SAUSSURE, Ferdinand de (1878): Mémoire sur le système primitif des voyelles dans les langues indo-européennes. Leipzig.

- (1983): Curso de Lingüistica General. Madrid: Alianza. De Mauro, ed.

- (1967): CLG. Wiesbaden: Harrassowitz. Engler, ed.

Sechehaye, Albert (1940): «Les trois linguistiques saussuriennes». Vox Romanica 5, $1-48$.

STROZIER, Robert (1985): «Saussure and the Intellectual Traditions of the Twentieth Century». Semiotica 57, 33-49.

Teso, Enrique del (1988): «Mensajes, entornos y anomalías». Contextos VI/12,45-73.

TYNIANOV-JAKOBSON (1928): «Problemas de los estudios literarios y lingüísticos», en (1976) Teoria de la literatura. Buenos Aires: Siglo XXI.

ZIPF, G. K. (1949): Human Behavior and the Principle of the Least Effort. Cambridge University Press. 